\title{
Direct diagnosis is superior to risk factor prediction tools for management of vessel wall disease
}

\author{
Afshin A. Divani ${ }^{1,2 *}$, Andreas R. Luft ${ }^{3}$, James D. Flaherty ${ }^{4}$ and Gundu H. R. Rao ${ }^{5}$ \\ 1 Department of Neurology, University of Minnesota, Minneapolis, MN, USA \\ 2 Department of Neurosurgery, University of Minnesota, Minneapolis, MN, USA \\ ${ }^{3}$ Department of Neurology, University of Zurich, Zurich, Switzerland \\ ${ }^{4}$ Division of Cardiology, Department of Medicine, Northwestern University, Chicago, IL, USA \\ ${ }^{5}$ Department of Laboratory Medicine and Pathology, University of Minnesota, Minneapolis, MN, USA \\ *Correspondence: divani@umn.edu
}

Diseases of the arteries cause more morbidity and mortality than most other noncommunicable diseases, including cancer. Atherosclerosis, the most prevalent vasculopathy, leads to myocardial infarction, stroke, or occlusions of peripheral arteries, but also causes slowly progressive disorders such as subcortical vascular encephalopathy or chronic kidney disease. A patient not presenting with a vascular event is typically assessed for atherosclerosis indirectly by cardiovascular risk factor prediction tools, rather than directly by imaging of the vessel wall for primary prevention (Goldstein et al., 2011). Only after a clinical event such as a stroke, the arteries are imaged by ultrasound, computed tomography (CT), magnetic resonance imaging (MRI), digital subtraction angiography (DSA). We suggest that, in primary prevention, a diagnostic paradigm that includes vascular imaging studies yields greater clinical benefits than assessing risk factors alone. Such an approach would provide an opportunity for customized therapy.

The Framingham study provided evidence supporting a causative role of risk factors, such as hypertension, diabetes, and altered blood lipid profiles, for vascular events (Dawber et al., 1951). Based on these results, management strategies for the control of these risk factors were developed (Dawber et al., 1951; Bitton and Gaziano, 2010). Risk monitoring tools currently available include the Framingham risk score (FRS) and the University of Minnesota 10 Point Risk Scoring System (Cohn et al., 2003). Imaging techniques, such as measuring intimal medium thickness (IMT) in carotid ultrasound, assessing coronary calcium content by CT, vessel volume measurements by MRI, and pulse wave velocity (PWV) index for monitoring arterial compliance are available to identify generalized (systemic) atherosclerosis and can be used to follow the progression of the disease (Rao and Sriram, 2010). Recent studies utilizing CT and MRI have demonstrated that the FRS may fail to identify up to $30 \%$ of individuals with atherosclerotic vascular disease (Johnson and Dowe, 2010).

One emerging technique to identify and track the progression (or regression) of carotid plaques is 3D ultrasound (Spence et al., 2002). Studies from the Robarts Research Institute in London, Ontario, have demonstrated that similar to total IMT measurements, total plaque volume (TPV), or total plaque area (TPA) measurements can be used to monitor the progression of atherosclerosis (Landry et al., 2004, 2005). Furthermore, these studies have demonstrated that aggressive lipid lowering using drugs such as atorvastatin can reduce the TPV significantly within 3 months. This technique has been shown to be sensitive enough to monitor diet or drug-induced changes in TPV. However, 3D ultrasound studies have also demonstrated that TPV may increase even after significant lowering of lipid levels, suggesting the need for additional therapies, such as antihypertensive therapy that has been shown to reduce plaque volume (Nissen et al., 2004). Although serial IMT measurement is more established, it is insensitive to drug-induced alterations in the TPV or TPA. Further studies of characterization of the morphology and composition of the plaque may enhance risk prediction.

For decades, the FRS has been considered the gold standard for cardiovascular risk prediction. Cohn et al. (2003) demonstrated that the University of Minnesota 10 Point Scale, which combines laboratory and physiologic measurements, is superior to the FRS at predicting clinical events over a 6-year follow-up. However, newer techniques

Table 1 | Risk factors for modeling versus disease identification strategy.

Risk factor identification Disease identification

PROS

Provides estimate of risk for cardiovascular events

Provides evidence for presence or absence of vascular disease

Usually simple and reproducible

Individuals without vascular disease not subjected to unnecessary treatment

Lower upfront cost

Individuals with vascular disease receive necessary treatment

Risk can be followed serially over time Disease progression or regression can be followed serially over time

\section{CONS}

Individuals without vascular disease may Requires imaging interpretation

receive unnecessary treatment*

Individuals with vascular disease may not Higher upfront and follow-up costs

receive necessary treatment

*This does not mean that vascular risk factors should not be treated in the absence of vascular disease. It means that the intensity of treatment (e.g., treatment goals of cholesterol) may be unnecessarily high in some patients. 
such as IMT and cardiac calcium scoring may replace indirect risk scoring systems. Recent 3D ultrasound techniques can monitor plaque volume, plaque progression, and plaque regression (Fenster et al., 2004, 2006; Landry et al., 2004, 2005, 2007; Ainsworth et al., 2005). Further studies on the morphology and composition of plaques will provide the ability to predict the nature of the plaque such as stability or vulnerability. There is an urgent need for the development of high-resolution, super-sensitive ultrasound systems that can monitor the atherosclerotic plaque in regional vascular beds of both small- and medium-sized vessels, so that the progression of the disease as well as the effectiveness of management of the disease with appropriate treatment modalities can be followed. We believe that the use of modern imaging techniques - especially of predilection sites of atherosclerosis, i.e., the carotids and the coronaries - as screening and monitoring tools in the primary prevention of vascular disease in addition to risk factor prediction could form the basis for a paradigm shift in vascular medicine from diagnosing risk factors to identifying the individual disease burden (see Table 1). However, such new techniques need to show their predictive value for vascular events and progression of chronic vascular disease before they can be uniformly recommended.

\section{REFERENCES}

Ainsworth, C. D., Blake, C. C., Tamayo, A., Beletsky, V., Fenster, A., and Spence, J. D. (2005). 3D ultrasound measurement of change in carotid plaque volume. Stroke 36, 1904-1909.

Bitton, A., and Gaziano, T. A. (2010). The framingham heart study's impact on global risk assessment. Prog. Cardiovasc. Dis. 53, 68-78.

Cohn, J. N., Hoke, L., Whitwam, W., Sommers, P. A., Taylor, A. L., Duprez, D., Roessler, R., and Florea, N. (2003). Screening for early detection of cardiovascular disease in asymptomatic individuals. Am. Heart J. 146, 679-685.

Dawber, T. R., Meadors, G. F., and Moore, F. E. Jr. (1951). Epidemiological approaches to heart disease: the framingham study. Am. J. Public Health Nations Health 41, 279-281.

Fenster, A., Blake, C., Gyacskov, I., Landry, A., and Spence, J.D. (2006). 3D ultrasound analysis of carotid plaque volume and surface morphology. Ultrasonics 44(Suppl. 1), e153-e157.

Fenster, A., Landry, A., Downey, D. B., Hegele, R. A. and Spence, J. D. (2004). 3D ultrasound imaging of the carotid arteries. Curr. Drug Targets Cardiovasc. Haematol. Disord. 4, 161-175.

Goldstein, L. B., Bushnell, C. D., Adams, R. J., Appel, L. J., Braun, L. T., Chaturvedi, S., Creager, M. A., Culebras, A., Eckel, R. H., Hart, R. G., Hinchey, J. A., Howard, V. J., Jauch, E. C., Levine, S. R., Meschia, J. F., Moore, W. S., Nixon, J.V., and Pearson, T.A. (2011). Guidelines for the primary prevention of stroke: a guideline for healthcare professionals from the American heart association/ American stroke association. Stroke 42, 517-584.

Johnson, K. M., and Dowe, D. A. (2010). The detection of any coronary calcium outperforms Framingham risk score as a first step in screening for coronary atherosclerosis. AJR Am. J. Roentgenol. 194, 1235-1243.
Landry, A., Ainsworth, C., Blake, C., Spence, J. D., and Fenster,A. (2007). Manual planimetric measurement of carotid plaque volume using three-dimensional ultrasound imaging. Med. Phys. 34, 1496-1505.

Landry, A., Spence, J. D., and Fenster, A. (2004). Measurement of carotid plaque volume by 3-dimensional ultrasound. Stroke 35, 864-869.

Landry, A., Spence, J. D., and Fenster, A. (2005). Quantification of carotid plaque volume measurements using 3D ultrasound imaging. Ultrasound Med. Biol. 31, 751-762.

Nissen, S. E., Tuzcu, E. M., Libby, P., Thompson, P. D., Ghali, M., Garza, D., Berman, L., Shi, H., Buebendorf, E., Topol, E. J., and Camelot Investigators. (2004). Effect of antihypertensive agents on cardiovascular events in patients with coronary disease and normal blood pressure. JAMA 292, 2217-2225.

Rao, G.H. R., and Sriram, V. (2010). "Management of vessel wall sisease is better than the management of risk factors," in 10th EUROCHAP Meetings of IUA, Paris. Spence, J. D., Eliasziw, M., DiCicco, M., Hackam, D. G., Galil, R., and Lohmann, T. (2002). Carotid plaque area: a tool for targeting and evaluating vascular preventive therapy. Stroke 33, 2916-2922.

Received: 16 December 2011; accepted: 24 February 2012; published online: 13 March 2012.

Citation: Divani AA, Luft AR, Flaherty JD and Rao GHR (2012) Direct diagnosis is superior to risk factor prediction tools for management of vessel wall disease. Front. Neur. 3:36. doi: 10.3389/fneur.2012.00036

This article was submitted to Frontiers in Endovascular and Interventional Neurology, a specialty of Frontiers in Neurology.

Copyright $\odot 2012$ Divani, Luft, Flaherty and Rao. This is an open-access article distributed under the terms of the Creative Commons Attribution Non Commercial License, which permits non-commercial use, distribution, and reproduction in other forums, provided the original authors and source are credited. 